\title{
Analysis on the Strengthening Buffer Operator Based on the Strictly Monotone Function
}

\author{
Hu Xiao-Li, Wu Zheng-Peng, and Han Ran
}

\begin{abstract}
We construct four kinds of new strengthening buffer operators by using inverse function theorem based on the axiom system of buffer operator. And demonstrate the GuanShi strengthening buffer operator which we compare with is a special case of our new operators. After studying the inner link and characteristics between the GuanShi and our new buffer operators, we greatly develop the application scope of strengthening buffer operator. This paper researches on buffer operators' construction with functions and gives a new direction for construction of buffer operators.
\end{abstract}

Index Terms-Strengthening buffer operator, grey system, monotone increasing function.

\section{INTRODUCTION}

Both for experimental or statistical data, we must analyze the data before modeling. Otherwise there will be not consistent between quantitative prediction and qualitative analysis conclusion. The crux of the problem doesn't depend on the advantages or disadvantages of the selected models. System behavior data is distorted by some external impact because of itself. Therefore, seeking combining site between quantitative forecast and qualitative analysis, trying to eliminate shock interference of system data, restoring the true data so as to improve the accuracy of prediction, is an important task for each prediction workers. Grey system theory is a kind of theory systems which find the law from the data itself. It seeks the change rule through original data mining and arrangement based on society, economy and ecology system and so on. Although the representation of objective system and data are complex, the data has a whole function. Therefore it contains some inherent law. The key lies in how to choose the appropriate approaches to mining and use it in the grey system theory. Liu SF proposed buffer operator theory which eliminate the impact of external shock interference successfully through the sequent ways of generation, weakening its randomness and showing its regularity of the data sequence [1]-[3]. And it could get the data sequence reflecting the changing regular patten of system.

Interference from shock disturbed factors to system behavior data sequence will speed up the development trend of data or make the amplitude wider. On the other hand, it

Manuscript received October 15, 2012; revised January 4, 2013. This work was supported in part by Communication University of China under Grant XNL1113.

The authors are with School of Science, Communication University of China, Beijing 100024, China (e-mail: huxiaoli@cuc.edu.cn, wuzhengpeng@126.com, hanran@cuc.edu.cn). also slows down the development trend of data or make data sequence of smaller amplitude. In order to exclude the interference of these factors, references [4]-[8] put forward a practical weakening buffer operator, and references [9]-[14] construct strengthening buffer operators. In this paper, we propose a new strengthening buffer operator and study its characteristics and internal relationship on the basis of the above work according to the buffer operator three axioms and thoughts of the average development rate of generalized time series. It is first time to research on buffer operators' construction with functions and gives a new direction for construction of buffer operators.

\section{COnStruction OF Strengthening Buffer OPERAtor}

Liu SF, Dang YG \& Guan YQ etc. construct strengthing buffer operator as follows. Set $X=(x(1), x(2), \cdots, x(n))$ to be a system data sequence. Set

$$
X D_{i}=\left(x(1) d_{i}, \cdots, x(n) d_{i}\right), i=1,2,3,4
$$

$$
\begin{gathered}
\text { among } x(k) d_{1}=\frac{x^{2}(k)}{\sqrt{\frac{x^{2}(k)+\cdots+x^{2}(n)}{n-k+1}}} \\
x(k) d_{2}=\frac{(n-k+1) x^{2}(k)}{x(k)+\cdots+x(n)} \\
x(k) d_{3}=\frac{x^{2}(k)}{[x(k) \times \cdots \times x(n)]^{n-k+1}} \\
x(k) d_{4}=\frac{x^{2}(k)}{\frac{n-k+1}{\frac{1}{x(k)}+\cdots+\frac{1}{x(n)}}} \\
(k=1,2, \cdots, n),
\end{gathered}
$$

Then when $X$ is a monotone increasing sequence, monotone attenuation or oscillation sequence, $D_{1}, D_{2}, D_{3}, D_{4}$ are strengthening buffer operators.

Theorem 1: Set $X=(x(1), x(2), \cdots, x(n))$ to be a non-negative system behavior data sequence, $x(i)>0$, then $x(k) d_{1} \leq x(k) d_{2} \leq x(k) d_{3} \leq x(k) d_{4} . \quad$ If $\quad$ and 
only $x(1)=x(2)=\cdots=x(n)$, all the equals sign was established.

Proof: see reference [13].

Here, we use the monotone function theory to construct a new strengthening buffer operator based on the strengthening buffer operators $D_{1}, D_{2}, D_{3}, D_{4}$.

Theorem 2: Set $X=(x(1), x(2), \cdots, x(n))$ to be a non-negative system behavior data sequence, and $x(i)>0, f>0$. $f$ is a strictly monotone increasing function whose inverse function is $g$. Among them

$$
x(k) e_{1}=g\left\{\frac{f^{2}(x(k))}{\sqrt{\frac{f^{2}(x(k))+\cdots+f^{2}(x(n))}{n-k+1}}}\right\}
$$

Set $X E_{1}=\left(x(1) e_{1}, \cdots x(n) e_{1}\right)$

Then when $X$ is a monotone increasing sequence, monotone attenuation sequence or oscillation sequence, $E_{1}$ is the strengthening buffer operator.

Proof: Easy to verify that

$$
x(n) e_{1}=g\left\{\frac{f^{2}(x(n))}{\sqrt{\frac{f^{2}(x(n))}{n-n+1}}}\right\}=x(n),
$$

So $E_{1}$ meets the buffer operator fixed point axiom. And it also meets the buffer operator axiom of fully using information and the analytic change, standardization axiom. So $E_{1}$ is a buffer operator.

Because $f$ is a strictly monotone increasing function,

1) If $X$ is a monotone increasing sequence, because of $0<x(k) \leq \cdots \leq x(n)$, so

$$
\begin{gathered}
0<f^{2}(x(k)) \leq \cdots \leq f^{2}(x(n)), \\
0<f^{2}(x(k)) \leq \frac{f^{2}(x(k))+\cdots+f^{2}(x(n))}{(n-k+1)} \\
0<f(x(k)) \leq \sqrt{\frac{f^{2}(x(k))+\cdots+f^{2}(x(n))}{(n-k+1)}} \\
0<\frac{f^{2}(x(k))}{\sqrt{\frac{f^{2}(x(k))+\cdots+f^{2}(x(n))}{n-k+1}}} \leq f(x(k)) \\
x(k) e_{1}=g\left\{\frac{f^{2}(x(k))}{\sqrt{\frac{f^{2}(x(k))+\cdots+f^{2}(x(n))}{n-k+1}}}\right\} \leq g(f(x(k)))=x(k)
\end{gathered}
$$

therefore $E_{1}$ is a strengthening buffer operator.
2) If $X$ is a monotone attenuation sequence, because of $x(k) \geq \cdots \geq x(n)>0$, we get

$$
\begin{gathered}
f^{2}(x(k)) \geq \cdots \geq f^{2}(x(n))>0, \\
f^{2}(x(k)) \geq \frac{f^{2}(x(k))+\cdots+f^{2}(x(n))}{(n-k+1)}>0 \\
f(x(k)) \geq \sqrt{\frac{f^{2}(x(k))+\cdots+f^{2}(x(n))}{(n-k+1)}}>0 \\
\frac{f^{2}(x(k))}{\sqrt{\frac{f^{2}(x(k))+\cdots+f^{2}(x(n))}{n-k+1}}} \geq f(x(k))>0
\end{gathered}
$$

$$
x(k) e_{1}=g\left\{\frac{f^{2}(x(k))}{\sqrt{\frac{f^{2}(x(k))+\cdots+f^{2}(x(n))}{n-k+1}}}\right\} \geq g(f(x(k)))=x(k)
$$

therefore $E_{1}$ is a strengthening buffer operator.

(c) if $X$ is an oscillation sequence set

$$
\begin{aligned}
& x(k)=\max _{1 \leq i \leq n} x(i), x(h)=\min _{1 \leq i \leq n} x(i), \\
& x(k) \geq x(k), \cdots, x(n) ; x(h) \leq x(h), \cdots, x(n), \\
& f^{2}(x(k)) \geq f^{2}(x(k)), \cdots, f^{2}(x(n))>0, \\
& 0<f^{2}(x(h)) \leq f^{2}(x(h)), \cdots, f^{2}(x(n)), \\
& f^{2}(x(k)) \geq \frac{f^{2}(x(k))+\cdots+f^{2}(x(n))}{(n-k+1)}>0 \\
& f(x(k)) \geq \sqrt{\frac{f^{2}(x(k))+\cdots+f^{2}(x(n))}{(n-k+1)}}>0 \\
& \frac{f^{2}(x(k))}{\sqrt{\frac{f^{2}(x(k))+\cdots+f^{2}(x(n))}{n-k+1}}} \geq f(x(k))>0 \\
& x(k) e_{1}=g\left\{\frac{f^{2}(x(k))}{\sqrt{\frac{f^{2}(x(k))+\cdots+f^{2}(x(n))}{n-k+1}}}\right\} \geq g(f(x(k)))=x(k) \\
& 0<f^{2}(x(\mathrm{~h})) \leq \frac{f^{2}(x(\mathrm{~h}))+\cdots+f^{2}(x(n))}{(n-h+1)} \\
& 0<f(x(\mathrm{~h})) \leq \sqrt{\frac{f^{2}(x(\mathrm{~h}))+\cdots+f^{2}(x(n))}{(n-h+1)}}
\end{aligned}
$$




$$
0<\frac{f^{2}(x(h))}{\sqrt{\frac{f^{2}(x(h))+\cdots+f^{2}(x(n))}{n-h+1}}} \leq f(x(\mathrm{~h}))
$$

$$
x(h) e_{1}=g\left\{\frac{f^{2}(x(h))}{\sqrt{\frac{f^{2}(x(h))+\cdots+f^{2}(x(n))}{n-h+1}}}\right\} \leq g(f(x(\mathrm{~h})))=x(\mathrm{~h})
$$

$$
\begin{aligned}
& \max _{1 \leq i \leq n} x(i) \leq \max _{1 \leq i \leq n} x(i) e_{1}, \\
& \min _{1 \leq i \leq n} x(i) \geq \min _{1 \leq i \leq n} x(i) e_{1},
\end{aligned}
$$

therefore $E_{1}$ is a strengthening buffer operator.

Theorem 3: Set $X=(x(1), x(2), \cdots, x(n))$ to be a non-negative system behavior data sequence, and $x(i)>0, f>0 . f$ is a strictly monotone increasing function whose inverse function is $g$.Among them

$$
x(k) e_{4}=g\left\{\frac{f^{2}(x(k))}{\frac{n-k+1}{\frac{1}{f(x(k))}+\cdots+\frac{1}{f(x(n))}}}\right\}
$$

Set $X E_{4}=\left(x(1) e_{4}, \cdots x(n) e_{4}\right)$,

If $X$ is a monotone increasing sequence, attenuation or oscillation sequence, $E_{4}$ is the strengthening buffer operator.

Proof: easy to verify

$$
x(n) e_{4}=g\left\{\frac{\left.\frac{f^{2}(x(n))}{\frac{n-n+1}{1}}\right\}}{\frac{1}{f(x(n))}}=g(f(x(n)))=x(n),\right.
$$

So $E_{4}$ meets buffer operator fixed point axiom. And it also meets the buffer operator axiom of fully using information and the analytic change, standardization axiom. So $E_{4}$ is a buffer operator.

Because $\mathrm{f}$ is a strictly monotone increasing function,

1) If $X$ is a monotone increasing sequence, because of

$$
\begin{gathered}
0<x(k) \leq \cdots \leq x(n), \text { so } \\
0<f(x(k)) \leq \cdots \leq f(x(n)), \\
\frac{1}{f(x(k))} \geq \cdots \geq \frac{1}{f(x(n))}>0 \\
0<\frac{1}{f(x(k))}+\cdots+\frac{1}{f(x(n))} \leq(n-k+1) \frac{1}{f(x(k))} \\
\frac{(n-k+1)}{\frac{1}{f(x(k))}+\cdots+\frac{1}{f(x(n))}} \geq f(x(k))>0 \\
0<\frac{f^{2}(x(k))}{\frac{n-k+1}{\frac{1}{f(x(k))}+\cdots+\frac{1}{f(x(n))}}} \leq f(x(k))
\end{gathered}
$$

$$
x(k) e_{4}=g\left\{\frac{f^{2}(x(k))}{\frac{n-k+1}{\frac{1}{f(x(k))}+\cdots+\frac{1}{f(x(n))}}}\right\} g(f(x(k)))=x(k),
$$

therefore $E_{4}$ is a strengthening buffer operator.

2) If $X$ is a monotone attenuation sequence, because of

$$
\begin{gathered}
0<x(k) \leq \cdots \leq x(n) \text {, so } f(x(k)) \geq \cdots \geq f(x(n))>0, \\
0<\frac{1}{f(x(k))} \leq \cdots \leq \frac{1}{f(x(n))} \\
\frac{1}{f(x(k))}+\cdots+\frac{1}{f(x(n))} \geq(n-k+1) \frac{1}{f(x(k))}>0
\end{gathered}
$$

$$
0<\frac{(n-k+1)}{\frac{1}{f(x(k))}+\cdots+\frac{1}{f(x(n))}} \leq f(x(k))
$$

$$
\frac{f^{2}(x(k))}{\frac{n-k+1}{\frac{1}{f(x(k))}+\cdots+\frac{1}{f(x(n))}}} \geq f(x(k))>0
$$

$$
x(k) e_{4}=g\left\{\frac{f^{2}(x(k))}{\frac{n-k+1}{\frac{1}{f(x(k))}+\cdots+\frac{1}{f(x(n))}}}\right\} g(f(x(k)))=x(k),
$$

therefore $E_{4}$ is a strengthening buffer operator.

3 ) if $X$ is an oscillation sequence, set

$$
\begin{gathered}
x(k)=\max _{1 \leq i \leq n} x(i), x(h)=\min _{1 \leq i \leq n} x(i), \\
x(k) \geq x(k), \cdots, x(n) ; x(h) \leq x(h), \cdots, x(n), \\
f(x(k)) \geq f(x(k)), \cdots, f(x(n))>0, \\
0<f(x(h)) \leq f(x(h)), \cdots, f(x(n)), \\
0<\frac{1}{f(x(k))} \leq \cdots \leq \frac{1}{f(x(n))} \\
\frac{1}{f(x(k))}+\cdots+\frac{1}{f(x(n))} \geq(n-k+1) \frac{1}{f(x(k))}>0 \\
x(k) e_{4}=g\left\{\frac{(n-k+1)}{\frac{1}{f(x(k))}+\cdots+\frac{1}{f(x(n))}} \leq f(x(k))\right. \\
\frac{\frac{1}{f(x(k))}+\cdots+\frac{1}{f(x(n))}}{\frac{f^{2}(x(k))}{n-k+1}} \geq g(f(x(k)))=x(k) \\
\frac{1}{f(x(k))}+\cdots+\frac{1}{f(x(n))} \\
\frac{\left.f^{2}(x)\right)}{n-k+1}(x(k))>0
\end{gathered}
$$




$$
\begin{aligned}
& \frac{1}{f(x(h))} \geq \cdots \geq \frac{1}{f(x(n))}>0 \\
& 0<\frac{1}{f(x(h))}+\cdots+\frac{1}{f(x(n))} \leq(n-h+1) \frac{1}{f(x(h))} \\
& \frac{(n-h+1)}{\frac{1}{f(x(h))}+\cdots+\frac{1}{f(x(n))}} \geq f(x(h))>0 \\
& 0<\frac{f^{2}(x(h))}{\frac{n-h+1}{\frac{1}{f(x(h))}+\cdots+\frac{1}{f(x(n))}}} \leq f(x(h)) \\
& \begin{array}{c}
x(h) e_{4}=g \\
\frac{\frac{f^{2}(x(h))}{\frac{n-h+1}{f(x(h))}+\cdots+\frac{1}{f(x(n))}}}{\frac{1}{f(x)}} \leq g(f(x(h)))=x(h) \\
\max _{1 \leq i \leq n} x(i) \leq \max _{1 \leq i \leq n} x(i) e_{4}, \\
\min _{1 \leq i \leq n} x(i) \geq \min _{1 \leq i \leq n} x(i) e_{4},
\end{array}
\end{aligned}
$$

therefore $E_{4}$ is a strengthening buffer operator.

Theorem 4: Set $X=(x(1), x(2), \cdots, x(n))$ to be a non-negative system behavior data sequence, and $x(i)>0, f>0 . f$ is a strictly monotone increasing function whose inverse function is $g$.Among them,

$$
x(k) e_{2}=g\left\{\frac{(n-k+1) f^{2}(x(k))}{f(x(k))+\cdots+f(x(n))}\right\}
$$

Set $X E_{2}=\left(x(1) e_{2}, \cdots x(n) e_{2}\right)$.

If $X$ is a monotone increasing sequence, attenuation or oscillation sequence, $E_{2}$ is the strengthening buffer operator.

Proof: see reference [14].

Theorem 5: Set $X=(x(1), x(2), \cdots, x(n))$ to be a non-negative system behavior data sequence, and $x(i)>0, f>0, f$ is a strictly monotone increasing function whose inverse function is $g$. Among them,

$$
x(k) e_{3}=g\left\{\frac{f^{2}(x(k))}{[f(x(k)) \times \cdots \times f(x(n))]^{\frac{1}{n-k+1}}}\right\}
$$

$$
\text { Set } X E_{3}=\left(x(1) e_{3}, \cdots x(n) e_{3}\right) \text {. }
$$

Then when $X$ is a monotone increasing sequence, monotone attenuation or oscillation sequence, $E_{3}$ is the strengthening buffer operator.

Proof: see reference [14].

Theorem 6: Set $X=(x(1), x(2), \cdots, x(n))$ to be a non-negative system behavior data sequence and $x(i)>0, f>0 . f$ is a strictly monotone increasing function whose inverse function is $g$. Then $x(k) e_{1} \leq x(k) e_{2} \leq x(k) e_{3} \leq x(k) e_{4}$.

If and only if $x(1)=x(2)=\cdots=x(n)$, all the equals sign was established.

Proof: see the theorem 1 and $g$ is strictly monotone increasing function, so we get the result.

The results above are also established for weight vector $w=\left(w_{1}, \cdots, w_{n}\right), w_{i}>0$. The deducing process is similar.

If $f(x)=g(x)=x$, buffer operators $E_{1}, E_{2}, E_{3}, E_{4}$ are the strengthening buffer operators separately in reference [4].

$D_{1}, D_{2}, D_{3}, D_{4}$ are the strengthening buffer operators. And $D_{1}, D_{2}, D_{3}, D_{4}$ are the special cases.

\section{APPLICATION}

We set the system data sequence based on the monthly sales from January to July of the new product in reference [13] (see Table I) to explain the application of the strengthening buffer operators in process of data modeling prediction. The unit of sales in the table is ten million yuan.

\begin{tabular}{lrrrrrrr}
\multicolumn{7}{c}{ TABLE I: MONTHLY SALES OF NEW PRODUCT } \\
\hline \hline Month & 1 & 2 & 3 & 4 & 5 & 6 & 7 \\
Sales & 60.8 & 62.6 & 65.7 & 70.4 & 77.4 & 86.7 & 96.8 \\
\hline \hline
\end{tabular}

According to the Table I, we calculated the growth rate of monthly sales separately were $2.96 \%, 4.95 \%, 7.12 \%, 9.94 \%$, $12.01 \%$ and $11.65 \%$. It is easy to see that the first half of them grew excessively slower than the latter. We eliminated the interference from the impacting disturbance factors to the original data sequence. Take $f(x)=g(x)=x$ to construct the buffer operator, and get the calculation results are as follows:

When no buffer operator to effect,

$$
\hat{x}(k+1)=693.919 e^{0.084 k}-693.119 ;
$$

When buffer operator $D_{2}$ to effect,

$$
\hat{x}(k+1)=385.738 e^{0.125 k}-333.378
$$

When buffer operator $D_{1}$ to effect,

$$
\hat{x}(k+1)=376.837 e^{0.127 k}-324.897
$$

TABLE II: GM $(1,1)$ MODEL IN THREe CoNDITIONS

\begin{tabular}{ccc}
\hline \hline Buffer Operator & Predicted Value & $\begin{array}{c}\text { Prediction } \\
\text { Relative Error }(\%)\end{array}$ \\
\hline Non & 92.62 & 4.5 \\
$D_{2}$ & 95.51 & 1.3 \\
$D_{1}$ & 95.71 & 1.1 \\
\hline \hline
\end{tabular}

Take $f(x)=x^{2}, g(x)=x^{0.5}$ to construct the buffer operator, and get the calculation results are as follows:

When buffer operator $E_{2}^{1}$ to effect,

$$
\hat{x}(k+1)=376.836 e^{0.1265 k}-324.897
$$

When buffer operator $E_{1}^{1}$ to effect,

$$
\hat{x}(k+1)=359.62 e^{0.13 k}-308.54
$$


TABLE III: GM(1,1) MODEL IN THREE CONDITIONS

\begin{tabular}{ccc}
\hline \hline Buffer Operator & Predicted Value & $\begin{array}{c}\text { Prediction } \\
\text { Relative Error(\%) }\end{array}$ \\
\hline Non & 92.62 & 4.5 \\
$E^{1}{ }_{2}$ & 95.71 & 1.1 \\
$E^{1}$ & 96.1 & 0.72 \\
\hline \hline
\end{tabular}

\section{CONCLUSION}

Based on the researches of others, sequence of the front part grow or decay too slowly, while the latter too quickly. It makes inconsistent results of quantitative prediction and qualitative analysis. The new strengthening buffer operators could solve the problems effectively in the process of constructing models to predict. In the process of constructing buffer operators, we used to construct one by one. This is the first time to connect function with buffer operators to construct one broad class of buffer operators. It supplies several choices to resolve the problems in shock data sequence modeling and gives the new direction to construct buffer operators by function. But research of how to optimize is underway.

\section{REFERENCES}

[1] S.-F. Liu, Y.-G. Dang and Z.-G. Fang, Grey System Theory And Its Application, Beijing: Science Press, 2004.

[2] S.-F. Liu, "The Three Axioms Of Buffer Operator And Their Applications," The Journal of Grey System, 1991, vol. 3, no. 1, pp. 39-48.

[3] S.-F. Liu, "Buffer Operator and Its Application," Grey System Theory And Practice, 1992, vol. 2, no. 1, pp. 45-50.

[4] S.-F. Liu, "Shock Disturbed System Prediction Trap And Buffer Operator," Journal Of Huazhong University Of Sci. \& Tech., 1997, vol. 25, no. 1, pp. 25-27.

[5] N.-M. Xie and S.-F.Liu, "A New Weakening Buffer Operator." Chinese Journal Of Management Science, 2003, vol. 11, pp. 46-48.

[6] Y.-G. Dang, S.-F. Liu, and B. Liu, "The Research On Weakening Buffer Operators," Chinese Journal Of Management Science, 2004, vol. 12, no. 2, pp. 108-111.

[7] Y.-Q. Guan and S.-F. Liu, "Study On Weakening Buffer Operator Sequence," Chinese Journal Of Management Science, 2007, vol. 15, no. 4, pp. 89-92.

[8] J. Cui and Y.-G. Dang, "A Kind Of New Weakening Buffer Operators Structure And Its Application," Control And Decision, 2008, vol. 23, no. 7, pp. 741-750.

[9] Y.-G. Dang, B. Liu, and Y.-Q. Guan, "The Research On Strengthening Buffer Operators," Control And Decision, 2005, vol. 20, no. 12, pp. 1332-1336.

[10] N.-M. Xie and S.-F. Liu, "Strengthening Buffer Operator's Characteristics And Some Practical Construction Of Strengthening Operator," Statistics and Decision, 2006, no. 4, pp. 9-10.

[11] Y.-Q. Guan and S.-F. Liu, "Study On Strengthening Buffer Operator Sequence And m Order Operator Role," Journal Of Yunnan Normal University, 2007, vol. 27, no. 1, pp. 32-35.

[12] Y.-G. Dang, S.-F. Liu, and C.-M. Mi, "Research On Strengthening Buffer Operators' Characteristics," Control And Decision, 2007, vol. 22, no. 7, pp. 730-734.

[13] Y.-Q. Guan and S.-F. Liu, "Based on the fixed point of strengthening buffer sequence operator and its application," Control And Decision, 2007, vol. 22, no. 10, pp. 1189-1192.

[14] Z.-P. Wu, S.-F. Liu, and C.-M. Mi, "Study On The Strengthening Buffer Operators Based On The Strictly Monotone Function," Journal of Grey System, 2008, vol. 11, no. 2, pp. 113-118.

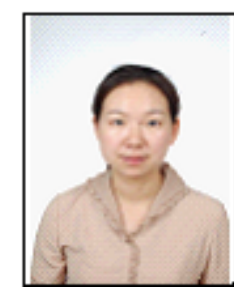

and Econometrics.
Hu Xiaoli was born on 4th October, 1978, in Yidu city, Hubei province. Now she is a $\mathrm{PhD}$ candidate majoring in Economics at Beijing Normal University. And also she is a lecturer at Communication University of China. She is a lecturer of Departmen of Statistics in School of Science at Communication University of China in Beijing from Sep. 2005 to present. She is interested in Financial Engineering 